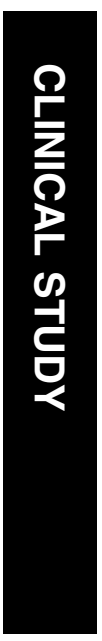

\section{Penetrating keratoplasty for bullous keratopathy after trabeculectomy}

${ }^{1}$ Department of Ophthalmology, University of Tokyo Graduate School of Medicine, Tokyo, Japan

${ }^{2}$ Inoue Eye Hospital, Tokyo, Japan

${ }^{3}$ Department of Corneal Tissue Regeneration, University of Tokyo Graduate School of Medicine, Tokyo, Japan

Correspondence: S Amano, Department of

Ophthalmology, University of Tokyo Graduate School of Medicine,

07-3-1 Hongo,

Bunkyo-ku,

Tokyo 113-8655, Japan

Tel: + 8135800 5109;

Fax: + 81356005109.

E-mail: amanos-tky@

umin.ac.jp

Received: 10 October 2007 Accepted in revised form: 28 February 2008

Published online: 28 March 2008

The authors have no commercial or proprietary interest in the products or companies mentioned in the current article.

\begin{abstract}
Purpose To evaluate the results of penetrating keratoplasty (PK) in eyes with bullous keratopathy having previously undergone trabeculectomy.

Methods We reviewed medical records of patients that had undergone PK for bullous keratopathy having previously undergone trabeculectomy. A total of 34 eyes in 34 patients were included in the study. As a control group, we reviewed the medical records of 26 eyes in 26 patients that had no history of glaucoma and who had undergone PK for bullous keratopathy in the same period at the same institutes. Kaplan-Meier survival analysis was performed to estimate the cumulative probability of successful intraocular pressure (IOP) control, immune reaction-free graft, and graft survival.

Results Visual acuity improved by more than two lines in 30-34 eyes (88\%). Log-rank tests showed a statistically significant difference in the cumulative probability of successful IOP control between eyes with functioning blebs and eyes with non-functioning blebs $(P=0.0005)$, but not between the experimental subjects of 34 eyes and the control group $(P=0.198)$. The log-rank test did not show statistically significant differences in the cumulative probability of immune reactionfree grafts and graft failure between the subjects and the control group and between eyes with functioning blebs and eyes with non-functioning blebs.

Conclusion The outcomes for PK in bullous keratopathy are good for patients having previously undergone trabeculectomy. Surgical procedures should be performed before PK in eyes with non-functioning blebs even when IOP is controllable with medication.
\end{abstract}

S Fukuoka', N Honda', T Usui', K Inoue², $S$ Yamagami ${ }^{3}$, M Araie $^{1}$ and S Amano'
Eye (2009) 23, 940-944; doi:10.1038/eye.2008.97; published online 28 March 2008

Keywords: penetrating keratoplasty; bullous keratopathy; trabeculectomy

\section{Introduction}

Bullous keratopathy can be caused by various diseases and surgeries, including Fuchs corneal endothelial dystrophy, iridocorneal endothelial dystrophy, cataract surgery, and glaucoma surgery. Trabeculectomy is one of these possible causes for bullous keratopathy. Both intraoperative insult to the corneal endothelium by surgical manoeuvres and anti-metabolites, as well as intra- and post-operative flat anterior chamber (leading to iris-cornea touching) may induce corneal endothelial dysfunction after trabeculectomy. In Japan, glaucoma surgery (including trabeculectomy) was the third most common cause of bullous keratopathy (5.3\%) following cataract surgery (44.4\%) and laser iridotomy (23.4\%). ${ }^{1}$ In eyes with bullous keratopathy having previously undergone trabeculectomy, the results of corneal transplantation may be affected by pre-existing glaucoma conditions and the presence of filtrating blebs. Alternatively, corneal transplantation may affect the control of intraocular pressure (IOP). In this regard, the results of corneal transplantation in eyes with bullous keratopathy having previously trabeculectomy are of great clinical interest. However, only a few studies have dealt with this matter. Kirkness et $a l^{2}$ evaluated the results of penetrating keratoplasty (PK) following trabeculectomy in 26 eyes and reported that there was a $45 \%$ probability of maintaining normal IOP with medication at 5 years after PK, 
and a similar probability for graft survival. In this study, we evaluated the results of PK in eyes with bullous keratoplasty having previously trabeculectomy, focusing on the influence of bleb presence on the results.

\section{Materials and methods}

We reviewed the medical records of patients that had undergone PK for bullous keratopathy having previously trabeculectomy between 1990 and 2005 at the University of Tokyo Graduate School of Medicine and the Inouye Eye Hospital. The subjects were excluded from the study if their follow-up period after PK was less than 1 year. A total of 34 eyes in 34 patients were included in the study. There were 19 eyes in 19 men and 15 eyes in 15 women. As a control group, we reviewed the medical records of 26 eyes in 26 patients that had no history of glaucoma and who underwent PK for bullous keratopathy in the same period at the same institutes. The demographic and clinical data are shown in Table 1 . This study was approved by the institutional review board of University of Tokyo Hospital and adhered to the tenets of the Declaration of Helsinki.

The data retrieved from patient medical records included: the type of glaucoma, previous cataract surgery, the number of previous trabeculectomy surgeries, bleb status, IOP control before and after PK, the number of anti-glaucoma medications, the presence of anterior synechia, surgical technique used, best spectacle-corrected visual acuity, the status of graft clarity, graft failure, and allograft immune reaction. The number of anti-glaucoma medications including eye drops and oral acetazolamide was scored in each eye. Use of oral acetazolamide of any doses was scored as one medication. Pre-operative number and highest number during 2 years after PK were compared between groups. A functioning bleb was defined as a cystic or diffuse filtrating bleb with a concurrent IOP of at least
$3 \mathrm{~mm} \mathrm{Hg}$ lower than the pre-operative level with no or fewer ocular hypotensive medications than preoperatively. ${ }^{3}$ PK was performed with 10-0 nylon continuous running sutures in 19 eyes and with interrupted sutures in 15 eyes. The donor size ranged from 7.5 to $8.5 \mathrm{~mm}$. The donor size was $0.25 \mathrm{~mm}$ oversized to the recipient bed size. Eye drops of $0.4 \%$ ofloxacine and $0.1 \%$ phosphate betamethasone were instilled six times daily during the month after surgery and their use was gradually tapered off. IOP was measured using a Goldmann's applanation tonometre. When IOP could not be measured accurately due to irregular ocular surface, IOP was measured using pneumatic tonometre.

Kaplan-Meier survival analysis was performed to estimate the cumulative probability of successful IOP control, immune reaction-free grafts, and graft survival. Successful control of IOP was defined as a reading below $21 \mathrm{~mm} \mathrm{Hg}$ with same or fewer ocular hypotensive medications than pre-operatively and no need for further glaucoma surgery. A corneal graft was defined as rejected when it became oedematous and showed signs of immunological reaction such as rejection line, infiltrations keratic precipitates, and anterior segment inflammation. Graft failure was defined as an irreversible loss of central graft clarity and was determined clinically using a slit-lamp biomicroscope. The log-rank test was used to compare the cumulative probability of the eyes of subjects after trabeculectomy with those of the control group, as well as eyes with functioning blebs and eyes with non-functioning blebs. The log-rank test was also used to compare the cumulative probability of eyes with running suture with eyes with interrupted suture. Unpaired $t$-test was used to compare IOP between groups. Mann-Whitney $U$-test was used to compare number of anti-glaucoma medications between groups. All data are reported here as mean \pm s.d. unless otherwise specified.

Table 1 Demographic and clinical data of the subjects and the control

\begin{tabular}{lll}
\hline & Subjects & Control \\
\hline Men : women & $19: 15$ & $15: 11$ \\
Age (range) (years) & $62.6 \pm 12.3(36-89)$ & $70.2 \pm 11.2(33-86)$ \\
Follow-up (range) (years) & $5.4 \pm 4.7(1-14)$ & $4.3 \pm 2.2(1-12)$ \\
Diagnosis for glaucoma & POAG nine eyes, PACG seven eyes, & - \\
Causes of bullous keratopathy & SOAG seven eyes, SACG five eyes, unknown six eyes & Cataract surgery 14 eyes \\
& & Corneal endotheliitis five eyes \\
PK surgery & & Sato's operation four eyes \\
& & Laser iridotomy three eyes \\
& PK alone 26 eyes & PK alone 19 eyes \\
& PK + cataract surgery eight eyes & PK + cataract surgery seven eyes
\end{tabular}

Abbreviations: PACG, primary angle closure glaucoma; POAG, primary open angle glaucoma; PK, penetrating keratoplasty; SACG, secondary angle closure glaucoma; SOAG, secondary open angle glaucoma. 


\section{Results}

The eyes had previously undergone trabeculectomy one to six times (mean, $2.5 \pm 1.7$ times) before PK. The period between the first trabeculectomy and PK ranged from 0.5 to 36.2 years (mean, $12.5 \pm 10.1$ years). The period between the last trabeculectomy and PK ranged from 0.3 to 33.4 years (mean, $8.2 \pm 8.2$ years). Twenty eyes (59\%) had previously undergone cataract surgery before PK.

Visual acuity improved by more than two lines in 30 of 34 eyes (88\%) and visual acuity stayed the same as preoperation in four eyes (Figure 1). In the control group, visual acuity improved by more than two lines in 25 of 26 eyes $(96 \%)$, and visual acuity stayed the same as preoperation in one eye.

A functioning filtering bleb was present in 21 of 34 eyes before PK. The remaining 13 eyes had flat and non-functioning blebs. All of the functional blebs were maintained for 1 year after PK. The mean IOP prior to PK and the highest IOP during the year after PK in each group are shown in Table 2. There was no statistically significant difference in IOP before PK between the subjects and the control eyes $(P=0.099)$ and between eyes with functioning vs non-functioning blebs $(P=0.0715)$. While there was no statistically significant difference between the highest IOP between the eyes of the subjects and of the controls $(P=0.494)$, there was a statistically significant difference in the highest IOP between eyes with a functioning bleb and nonfunctioning blebs $(P=0.0119)$. The changes of the number of anti-glaucoma medications in each group are shown in Table 3 . There were statistically significant differences in the number of anti-glaucoma medications

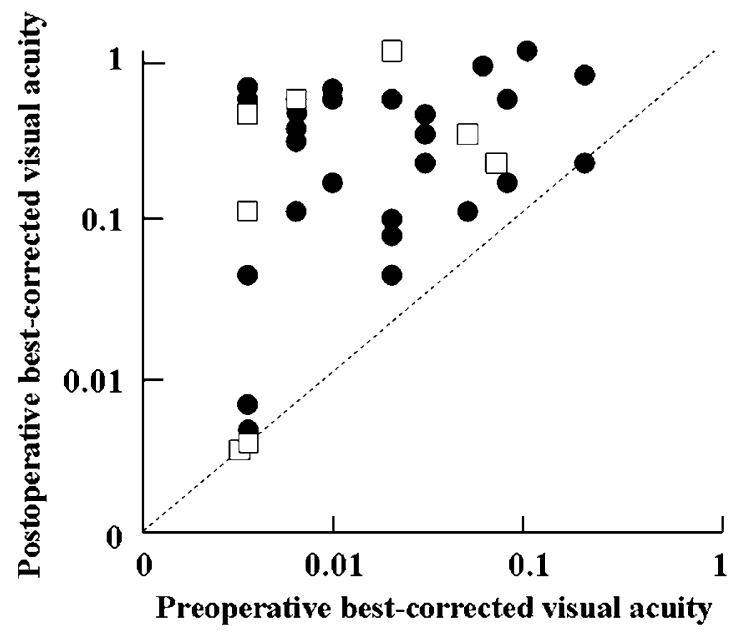

Figure 1 Relation between pre-operative visual acuity and visual acuity after penetrating keratoplasty (PK). Circles indicate eyes that underwent PK alone. Squares indicate eyes that underwent PK and cataract surgery simultaneously. between eyes with a functioning bleb and non-functioning blebs pre-operatively $(P=0.0095)$ and during 2 years after PK $(P=0.0265)$.

Kaplan-Meier survival analysis showed that the cumulative probability of successful IOP control after 2 years in the subjects (34 eyes), the control group, eyes with functioning blebs, and eyes with non-functioning bleb were 35.3, 40.9, 57.1, and 0\%, respectively (Figure 2). Log-rank tests showed a statistically significant difference in the cumulative probability of successful IOP control between eyes with a functioning bleb and eyes with non-functioning blebs $(P=0.0005)$, but not between the subjects and the control group $(P=0.198)$.

In total, 7 (54\%) out of 13 eyes with non-functioning bleb required surgical intervention to decrease IOP within 2 years after PK. Trabeculectomy was performed in five patients and revision by needling the bleb was performed in one patient. One patient was treated with cyclophotocoagulation. On the other hand, 3 out of 21 eyes $(14 \%)$ with functioning blebs needed needling of the bleb to decrease IOP within 2 years after PK.

Kaplan-Meier survival analysis showed that the cumulative probability of immune reaction-free grafts at 2 years in the subjects, the control group, eyes with

Table 2 Data of mean intraocular pressure in each group

\begin{tabular}{lcc}
\hline & Pre-operation & $\begin{array}{c}\text { Highest IOP } \\
\text { during the } \\
\text { year after PK }\end{array}$ \\
\hline All subjects (34 eyes) & $12.7 \pm 5.4$ & $24.4 \pm 9.2$ \\
Control (26 eyes) & $14.8 \pm 3.5$ & $\begin{array}{c}(92 \% \text { increase) } \\
22.4 \pm 7.9\end{array}$ \\
$\begin{array}{l}\text { Eyes with functioning } \\
\text { bleb (21 eyes) }\end{array}$ & $11.3 \pm 4.1$ & $\begin{array}{c}51 \% \text { increase) } \\
\text { Eyes with non-functioning } \\
\text { bleb (13 eyes) }\end{array}$ \\
\hline
\end{tabular}

Abbreviations: IOP, intraocular pressure; PK, penetrating keratoplasty. Mean \pm standard deviation of IOP in $\mathrm{mmHg}$.

Table 3 Mean number of anti-glaucoma medications in each group

\begin{tabular}{lcc}
\hline & Pre-operation & $\begin{array}{c}\text { Highest number } \\
\text { during 2 years } \\
\text { after PK }\end{array}$ \\
\hline All subjects (34 eyes) & $0.88 \pm 0.93$ & $1.84 \pm 1.52$ \\
$\begin{array}{l}\text { Control (26 eyes) } \\
\text { Eyes with functioning }\end{array}$ & 0 & $0.62 \pm 1.04$ \\
bleb (21 eyes) & $0.50 \pm 0.69$ & $1.43 \pm 1.47$ \\
$\begin{array}{l}\text { Eyes with non-functioning } \\
\text { bleb (13 eyes) }\end{array}$ & $1.25 \pm 0.75$ & $2.64 \pm 1.43$ \\
\hline
\end{tabular}

Abbreviation: PK, penetrating keratoplasty. 

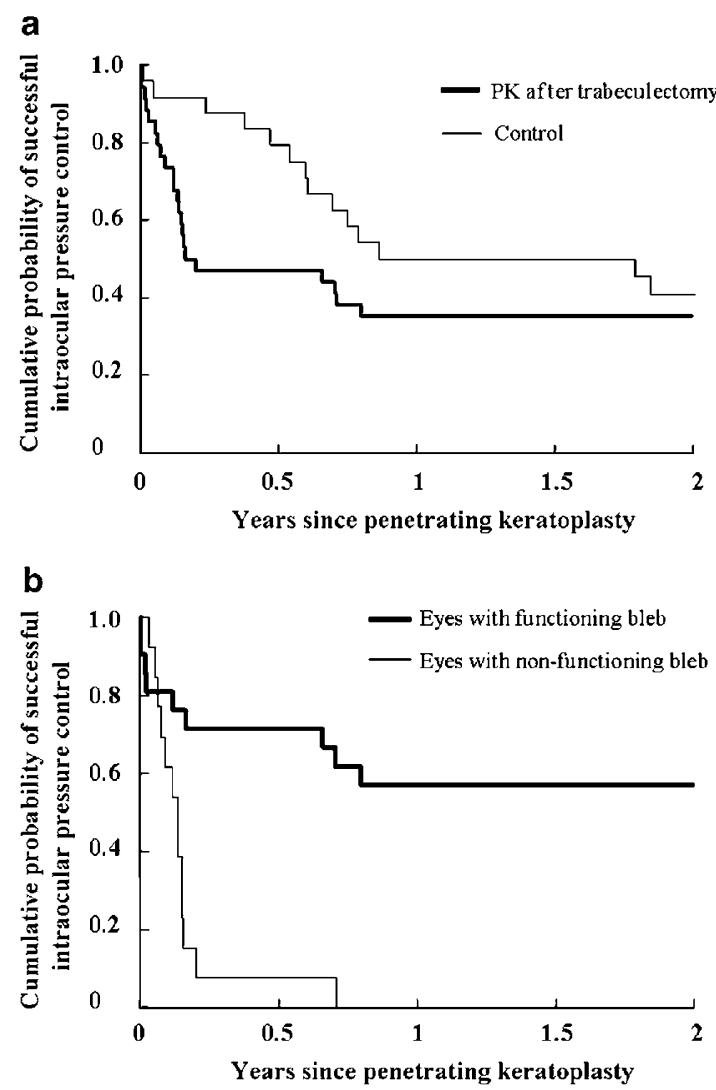

Figure 2 Kaplan-Meier analysis of the cumulative probability of successful intraocular pressure (IOP) control after penetrating keratoplasty. The cumulative probability of successful IOP control was not significantly different between the subjects (34 eyes) and the control group (a) but was significantly different between eyes with functioning blebs and eyes with nonfunctioning blebs (b). Log-rank tests showed a statistically significant difference between eyes with a functioning bleb and eyes with non-functioning blebs $(P=0.0005)$, but not between the subjects and the control group $(P=0.198)$.

functioning blebs, and eyes with non-functioning blebs was $78.2,80.8,69.2$, and $92.3 \%$, respectively. The log-rank test did not show a statistically significant difference in the cumulative probability of immune reaction-free grafts between the subjects and the control group $(P=0.881)$ and between eyes with functioning blebs and eyes with non-functioning blebs $(P=0.156)$.

Kaplan-Meier survival analysis also showed that the cumulative probability of graft survival at 2 years in the subjects, control group, eyes with functioning bleb, and eyes with non-functioning blebs was 62.1, 73.1, 64.1, and $59.2 \%$, respectively. The log-rank test did not show a statistically significant difference in the cumulative probability of graft survival between the subjects and the control group $(P=0.360)$, and between eyes with functioning blebs and eyes with non-functioning blebs $(P=0.787)$.
The cumulative probability of immune reaction-free grafts at 2 years in 19 eyes with running suture and 15 eyes with interrupted suture was 83.5 and $71.8 \%$, respectively ( $P=0.496$, the log-rank test). The cumulative probability of graft survival at 2 years in eyes with running suture and eyes with interrupted suture was 78.2 and $43.6 \%$, respectively ( $P=0.101$, the log-rank test). The suture technique did not have influence on postoperative outcomes.

\section{Discussion}

Visual acuity improved by more than two lines in $88 \%$ of the eyes after PK, suggesting that PK is effective at restoring visual function in eyes with bullous keratopathy having previously trabeculectomy. Visual acuity stayed hand motion due to advanced progression of glaucoma in four eyes, and due to unknown causes in one eye. Although detailed examination of the visual field is sometimes difficult in cases with advanced bullous keratopathy, patients with advanced glaucoma should be informed before PK that their visual acuity may possibly remain at pre-operative levels.

The cumulative probability of successful IOP control at 2 years after PK was lower in the eyes after trabeculectomy than in the control group, although not statistically significant. Although all blebs were maintained for 1 year after PK in eyes with functioning blebs after trabeculectomy, the success rate of IOP control gradually decreased within 1 year in these eyes. Even when filtrating blebs seemed to be maintained, sutures and post-operative inflammation might have decreased the size of the blebs or occluded the angle further. In eyes with non-functioning blebs, IOP control after PK was even worse. IOP control failed in all eyes with nonfunctioning blebs and $54 \%$ of eyes required surgical intervention to decrease IOP within 2 years after PK. Even when IOP is under control with medication in eyes with non-functioning blebs, IOP control may easily fail with surgeries or topical steroids. Taken together, these results indicate that PK very likely induces failure of IOP control in eyes with non-functioning blebs, and thus it is desirable that surgical procedures, such as needling of the bleb and re-trabeculectomy, be performed in those eyes before PK, even if IOP is under control with medication.

The occurrence rate of glaucoma in eyes after PK is estimated at 20-30\%. ${ }^{4}$ The causes of glaucoma include progression of the synechial angle closure and response to topical steroids. These mechanisms should also work in eyes after PK for bullous keratopathy having previously trabeculectomy. Descemet's stripping automated endothelial keratoplasty (DSAEK) should require less topical steroid and induce less synechial 
angle closure after surgery than PK because DSAEK is not an open-sky procedure and is less invasive than PK. Thus, DSAEK may have an advantage over PK in regard to post-operative glaucoma control.

The cumulative probability of immune reaction-free grafts at 2 years after PK was similar in eyes having previously trabeculectomy and in control eyes. On the other hand, the immune reaction-free graft rate in eyes with functioning blebs was lower than that in eyes with no-functioning bleb, although not statistically significant. The presence of blebs might have facilitated traffic between the anterior chamber and the surrounding lymphoid tissues, thereby facilitating the recognition of alloantigen in corneal grafts.

The cumulative probability of graft survival at 2 years after PK was similar in the eyes having previously trabeculectomy and in control eyes. This result suggests that the history of trabeculectomy has little influence on graft survival after PK. On the other hand, several studies reported that glaucoma history is one of the risk factors for graft failure after PK. ${ }^{5-8}$ Reinhard et $a l^{8}$ reported that the estimated 3-year graft survival rate was $71 \%$ in eyes with a glaucoma history in contrast to $89 \%$ in eyes without glaucoma history, and that there was no difference with respect to immune reaction between eyes with or without glaucoma history. Hence, they concluded that glaucoma history affects graft prognosis negatively, presumably through the negative influence of post-operatively-elevated IOP on a vulnerable graft endothelium, and not by an immune reaction. However, because they did not show the data on the post-operative IOP in each group, the exact influence of post-operative IOP on graft survival may not be evaluated with their data. Moreover, several studies reported that increased IOP has little effect on the corneal endothelium., ${ }^{9,10}$ Taken together, our results showing that the history of trabeculectomy had little effect on graft survival in spite of poor post-operative IOP control are not surprising.

In conclusion, PK for bullous keratopathy having previously trabeculectomy reduces IOP control, particularly in eyes with non-functioning blebs. The rates of immune reaction-free grafts and graft survival after PK for bullous keratopathy having previously trabeculectomy are similar to those after PK for bullous keratopathy in eyes without glaucoma. Thus, the outcomes for PK in bullous keratopathy are good for patients having previously undergone trabeculectomy surgery, but it may be desirable that surgical procedures such as needling of the bleb and re-trabeculectomy be performed before PK in eyes with non-functioning blebs, even when IOP is under control with medication.

\section{References}

1 Shimazaki J, Amano S, Uno T, Maeda N, Yokoi N, the Japan Bullous Keratopathy Study Group. National Survey on Bullous Keratopathy in Japan. Cornea 2007; 26: 274-278.

2 Kirkness CM, Steele AD, Ficker LA, Rice NS. Coexistent corneal disease and glaucoma managed by either drainage surgery and subsequent keratoplasty or combined drainage surgery and penetrating keratoplasty. Br J Ophthalmol 1992; 76: $146-152$.

3 Matsuo H, Tomidokoro A, Suzuki Y, Shirato S, Araie M. Late-onset transconjunctival oozing and point leak of aqueous humor from filtering bleb after trabeculectomy. Am J Ophthalmol 2002; 133: 456-462.

4 Ayyala RS. Penetrating keratoplasty and glaucoma. Surv Ophthalmol 2000; 45: 91-105.

5 Maguire MG, Stark WJ, Gottsch JD, Stulting RD, Sugar A, Fink NE et al. Risk factors for corneal graft failure and rejection in the collaborative corneal transplantation studies. Collaborative Corneal Transplantation Studies Research Group. Ophthalmology 1994; 101: 1536-1547.

6 Boisjoly HM, Tourigny R, Bazin R, Laughrea PA, Dubé I, Chamberland $\mathrm{G}$ et al. Risk factors of corneal graft failure. Ophthalmology 1993; 100: 1728-1735.

7 Yamagami S, Suzuki Y, Tsuru T. Risk factors for graft failure in penetrating keratoplasty. Acta Ophthalmol Scand 1996; 74: 584-588.

8 Reinhard T, Kallmann C, Cepin A, Godehardt E, Sundmacher R. The influence of glaucoma history on graft survival after penetrating keratoplasty. Graefes Arch Clin Exp Ophthalmol 1997; 235: 553-557.

9 Korey M, Gieser D, Kass MA, Waltman SR, Gordon M, Becker B. Central corneal endothelial cell density and central corneal thickness in ocular hypertension and primary openangle glaucoma. Am J Ophthalmol 1982; 94: 610-616.

10 Mortensen AC, Sperling S. Human corneal endothelial cell density after an in vitro imitation of elevated intraocular pressure. Acta Ophthalmol (Copenh) 1982; 60: 475-479. 